\title{
Dynamics of a predator-prey model with impulsive biological control and unilaterally impulsive diffusion
}

\author{
Jianjun Jiao', Shaohong Cai' , Limei Li ${ }^{2}$ and Yujuan Zhang ${ }^{3 *}$
}

\author{
"Correspondence: \\ zhangyujuan1968@163.com \\ ${ }^{3}$ College of Mathematics and \\ Information Science, Anshan \\ Normal University, Anshan, 114007, \\ P.R. China \\ Full list of author information is \\ available at the end of the article
}

\begin{abstract}
In this paper, we establish a predator-prey model with impulsive biological control and unilaterally impulsive diffusion. This predator-prey model for two regions, which are connected by diffusion of predator population, portrays the evolution of the population. We study the model for biological pest control in which a pest population is controlled by a program of periodic releases of a fixed yield of predators that prey on the pest population. We prove that there exists a globally asymptotically stable prey-extinction boundary periodic solution. The condition for permanence is also obtained. Simulations are also employed to verify our results. We conclude that the impulsive diffusion and releasing predator provide reliable tactic basis for pest management.
\end{abstract}

Keywords: predator-prey model; unilaterally impulsive diffusion; impulsive biological control; extinction; permanence

\section{Introduction}

The warfare between humans and pests has persisted for thousands of years. In the past few decades, man has adopted some advanced and modern weapons for instance chemical pesticides, biological pesticides, remote sensing and measure, computers, atomic energy etc. Some brilliant achievements have been obtained. However, the warfare will never be over. Although a great deal of pesticides were used to control pests, the insect pests impairing crops are increasing because of the resistance to the pesticide. With pesticides employed, the residual pests breed a large number of pests with resistance to pesticides. So the pesticide is invalid in a sense. Moreover, insect pests will remain. On the other hand, the chemical pesticides kill not only pests but also their natural enemies. Therefore, insect pests are rampant again. Then the effect of chemical control was challenged. Furthermore, the practice proves that long-term adopting chemical control may give rise to disastrous results, for example, we witness environmental contamination and toxicosis of man and animal, and so on.

The use of a natural enemy to suppress pests is one of the most important approaches in pest control. Biological control [1-8] is one of the reductions in pest populations from the actions of other living organisms, often called natural enemies or beneficial species. It is the purposeful introduction and establishment of one or more natural enemies from the

(c) 2016 Jiao et al. This article is distributed under the terms of the Creative Commons Attribution 4.0 International License (http://creativecommons.org/licenses/by/4.0/), which permits unrestricted use, distribution, and reproduction in any medium, provided you give appropriate credit to the original author(s) and the source, provide a link to the Creative Commons license, and indicate if changes were made. 
region of origin of an exotic pest, specifically for the purpose of suppressing the abundance of the pest in a new target region to a level at which it no longer causes economic damage. Jiao et al. [9] analyzed the dynamics of a stage-structured Holling mass defence predatorprey model with impulsive perturbations on predators,

$$
\left\{\begin{array}{l}
\frac{d x_{1}(t)}{d t}=r x_{2}(t)-r e^{-w \tau_{1}} x_{2}\left(t-\tau_{1}\right)-w x_{1}(t), \\
\frac{d x_{2}(t)}{d t}=r e^{-w \tau_{1}} x_{2}\left(t-\tau_{1}\right)-\frac{\beta x_{2}(t)}{1+a x_{2}+b x_{2}^{2}} x_{3}(t)-d_{3} x_{2}(t)-d_{4} x_{2}^{2}(t), \\
\frac{d x_{3}(t)}{d t}=\frac{k \beta x_{2}(t)}{1+a x_{2}+b x_{2}^{2}} x_{3}(t)-d x_{3}(t), \\
\triangle x_{1}(t)=0, \\
\triangle x_{2}(t)=0, \quad t=n \tau, n=1,2, \ldots, \\
\triangle x_{3}(t)=\mu, \\
\left(\varphi_{1}(\zeta), \varphi_{2}(\zeta), \varphi_{3}(\zeta)\right) \in C_{+}=C\left(\left[-\tau_{1}, 0\right], R_{+}^{3}\right), \quad \varphi_{i}(0)>0, i=1,2,3
\end{array}\right.
$$

where $x_{1}(t), x_{2}(t)$ represent the immature and mature pest densities, respectively. $x_{3}(t)$ denotes the density of nature enemy. The biological meanings of the parameters can be found in [9].

The dispersal is a ubiquitous phenomenon in the natural world. It is important for us to understand the ecological and evolutionary dynamics of populations mirrored by the large number of mathematical models devoted to it in the scientific literature [10-13]. In recent years, the analysis of these models focused on the coexistence of populations and the local (or global) stability of the equilibria [14-20]. Spatial factors play a fundamental role on the persistence and stability of the population, although the complete results have not yet been obtained even in the simplest one-species case. If the population dynamics with the effects of spatial heterogeneity is modeled by a diffusion process, most previous papers focused on the population dynamical system modeled by the ordinary differential equations. But in practice, it is often the case that diffusion occurs in a regular pulse. For example, when winter comes, birds will migrate between patches in search for a better environment, whereas they do not diffuse in other seasons, and the excursion of foliage seeds occurs at a fixed period of time every year. Thus impulsive diffusion provides a more natural description. Lately theories of impulsive differential equations [21] have been introduced into population dynamics. Jiao et al. [22] proposed and investigated the dynamical behaviors of a stage-structured predator-prey model with prey impulsively diffusing between two patches

$$
\left\{\begin{array}{l}
\frac{d x_{1}(t)}{d t}=x_{1}(t)\left(a_{1}-b_{1} x_{1}(t)\right), \\
\frac{d x_{2}(t)}{d t}=x_{2}(t)\left(a_{2}-b_{2} x_{2}(t)\right)-\alpha x_{2}(t) y_{2}(t), \\
\frac{d y_{1}(t)}{d t}=k \alpha x_{2}(t) y_{2}(t)-k \alpha e^{-w \tau_{1}} x_{2}\left(t-\tau_{1}\right) y_{2}\left(t-\tau_{1}\right)-w y_{1}(t), \\
\frac{d y_{2}(t)}{d t}=k \alpha e^{-w \tau_{1}} x_{2}\left(t-\tau_{1}\right) y_{2}\left(t-\tau_{1}\right)-d y_{2}(t), \\
\triangle x_{1}(t)=d_{1}\left(x_{2}(t)-x_{1}(t)\right), \\
\triangle x_{2}(t)=d_{2}\left(x_{1}(t)-x_{2}(t)\right), \\
\triangle y_{1}(t)=0, \\
\triangle y_{2}(t)=0,
\end{array}\right\} \quad t=n \tau, n=1,2, \ldots,
$$

where we suppose that the system is composed of two patches connected by diffusion and occupied by a single species $x_{i}(i=1,2)$ is the density of species in the $i$ th patch. $y_{1}(t)$, 
$y_{2}(t)$ represent the densities of the immature individual predator and mature individual predator at time $t$ in the second patch. The biological meanings of the parameters can be found in [22].

Theories of impulsive differential equations have been introduced into population dynamics lately [23-28]. Impulsive equations are found in almost every domain of applied science and have been studied in many investigations [28-33], they generally describe phenomena which are subject to steep or instantaneous changes. The theories of population dynamical systems and their applications have achieved many good results. However, the oasis vegetation degradation combined with a dynamical system has been considered very little. In this paper, we will investigate an impulsive dispersal on SIR model on restricting infected individuals boarding transports. We expect to obtain some dynamical properties of the investigated system. We also expect that impulsive dispersal will provide a reliable tactic for controlling epidemic.

The organization of this paper is as follows. In the next section, we introduce the model and background concepts. In Section 3, some important lemmas are presented. We give the globally asymptotically stable conditions of the prey-extinction boundary periodic solution of System (2.1), and the permanent condition of System (2.1). In Section 4 is a simulation analysis, and a brief discussion are given in the last section to conclude this work.

\section{The model}

In this paper, we establish a predator-prey model with impulsive biological control and unilaterally impulsive diffusion.

$$
\left\{\begin{array}{l}
\frac{d x_{1}(t)}{d t}=x_{1}(t)\left(a_{1}-b_{1} x_{1}(t)\right)-\beta_{1} x_{1}(t) y_{1}(t), \\
\frac{d y_{1}(t)}{d t}=k_{1} \beta_{1} x_{1}(t) y_{1}(t)-d_{1} y_{1}(t), \\
\frac{d y_{2}(t)}{d t}=y_{2}(t)\left(a_{2}-b_{2} y_{2}(t)\right), \\
\triangle x_{1}(t)=0 \\
\triangle y_{1}(t)=D y_{2}(t), \\
\triangle y_{2}(t)=-D y_{2}(t), \\
\triangle x_{1}(t)=0, \\
\triangle y_{1}(t)=\mu, \\
\triangle y_{2}(t)=0,
\end{array}\right\} \quad t=(n+l) \tau, n \in Z^{+},
$$

where we suppose that the system is composed of two patches connected by diffusion. These two patches are separated by rivers or highways or railways. The predator population can transverse the rivers or highways or railways, while the prey population cannot. $x_{1}(t)$ and $y_{1}(t)$ represent the numbers of preys and predators in the populations in patch 1 at time $t . y_{2}(t)$ represents the number of predators in the population in patch 2 at time $t . a_{1}>0$ represents the intrinsic growth rate of the prey population in patch 1. $b_{1}>0$ represents the coefficient of the intraspecific competition of the prey population in patch $1 . a_{2}>0$ represents the intrinsic growth rate of the predator population in patch 2. $b_{2}>0$ represents the coefficient of the intraspecific competition of the predator population in patch 2 . The predator consumes the prey according to $\beta_{1} x_{1}(t)$, in patch 1 at time $t . k_{1}$ is the rate of conversion of nutrients into the reproduction of the predator in patch $1 . d_{1}$ represents the death rate of the predator population in patch 1 . The pulse diffusion occurs every $\tau$ period ( $\tau$ is a positive constant), the system evolves from 
its initial state without being further affected by diffusion until the next pulse appears; $\Delta y_{i}((n+l) \tau)=y_{i}\left((n+l) \tau^{+}\right)-y_{i}((n+l) \tau)$ where $y_{i}\left((n+l) \tau^{+}\right)$represents the density of population in the $i$ th patch immediately after the $n$th diffusion pulse at time $t=(n+l) \tau$, while $y_{i}((n+l) \tau)$ represents the density of population in the $i$ th patch before the $n$th diffusion pulse at time $t=(n+l) \tau, 0<l<1, n \in Z_{+} .0<D<1$ represents the diffusive rate between two patches. $\Delta y_{1}((n+1) \tau)=y_{1}\left((n+1) \tau^{+}\right)-y_{1}((n+1) \tau)$ and $\mu$ represent the releasing amount of predator population at $t=(n+1) \tau, n \in Z_{+}$, in patch 1 .

\section{The lemmas}

The solution of (2.1), denoted by $X(t)=\left(x_{1}(t), y_{1}(t), y_{2}(t)\right)^{T}$, is a piecewise continuous function $X: R_{+} \rightarrow R_{+}^{3}, X(t)$ is continuous on $(n \tau,(n+l) \tau]$ and $((n+l) \tau,(n+1) \tau], n \in Z_{+}$and $X\left(n \tau^{+}\right)=\lim _{t \rightarrow n \tau^{+}} X(t), X\left((n+l) \tau^{+}\right)=\lim _{t \rightarrow(n+l) \tau^{+}} X(t)$ exist. Obviously the global existence and uniqueness of solutions of (2.1) is guaranteed by the smoothness properties of $f$, which denotes the mapping defined by right side of System (2.1) [21].

Let $V: R_{+} \times R_{+}^{3} \rightarrow R_{+}$, then $V$ is said to belong to class $V_{0}$, if:

(i) $V$ is continuous in $(n \tau,(n+l) \tau] \times R_{+}^{3}$ and $((n+l) \tau,(n+1) \tau] \times R_{+}^{3}$, for each $z \in R_{+}^{3}$, $n \in Z_{+}, V\left(n \tau^{+}, z\right)=\lim _{(t, y) \rightarrow\left(n \tau^{+}, z\right)} V(t, y), V\left((n+l) \tau^{+}, z\right)=\lim _{(t, y) \rightarrow\left((n+l) \tau^{+}, y\right)} V(t, y)$ exist.

(ii) $V$ is locally Lipschitzian in $z$.

Definition 3.1 $V \in V_{0}$, then, for $(t, z) \in(n \tau,(n+l) \tau] \times R_{+}^{3}$ and $((n+l) \tau,(n+1) \tau] \times R_{+}^{3}$, the upper right derivative of $V(t, z)$ with respect to the impulsive differential System (2.1) is defined as

$$
D^{+} V(t, z)=\limsup _{h \rightarrow 0} \frac{1}{h}[V(t+h, z+h f(t, z))-V(t, z)] .
$$

Since $\frac{d x_{i}(t)}{d t}=0$, when $x_{i}(t)=0 ; \frac{d y_{i}(t)}{d t}=0$, when $y_{i}(t)=0$, and $\Delta y_{1}(t)=\mu>0$, when $t=$ $(n+1) \tau$, we can easily obtain the following lemma.

Lemma 3.2 Suppose $X(t)$ is a solution of (2.1) with $X\left(0^{+}\right) \geq 0$, then $X(t) \geq 0$ for $t \geq 0$. Furthermore, $X(t)>0(t \geq 0)$ for $X\left(0^{+}\right)>0$.

Lemma 3.3 [21] Let the function $m \in P C^{\prime}\left[R^{+}, R\right]$ satisfy the inequalities

$$
\begin{cases}m^{\prime}(t) \leq p(t) m(t)+q(t), & t \geq t_{0}, t \neq t_{k}, k=1,2, \ldots, \\ m\left(t_{k}^{+}\right) \leq d_{k} m\left(t_{k}\right)+b_{k}, & t=t_{k},\end{cases}
$$

where $p, q \in P C\left[R^{+}, R\right]$ and $d_{k} \geq 0, b_{k}$ are constants; then

$$
\begin{aligned}
m(t) \leq & m\left(t_{0}\right) \prod_{t_{0}<t_{k}<t} d_{k} \exp \left(\int_{t_{0}}^{t} p(s) d s\right) \\
& +\sum_{t_{0}<t_{k}<t}\left(\prod_{t_{k}<t_{j}<t} d_{j} \exp \left(\int_{t_{0}}^{t} p(s) d s\right)\right) b_{k} \\
& +\int_{t_{0}}^{t} \prod_{s<t_{k}<t} d_{k} \exp \left(\int_{s}^{t} p(\sigma) d \sigma\right) q(s) d s, \quad t \geq t_{0} .
\end{aligned}
$$

Now, we show that all solutions of (2.1) are uniformly ultimately bounded. 
Lemma 3.4 There exists a constant $M>0$ such that $x_{1}(t) \leq M, y_{1}(t) \leq M, y_{2}(t) \leq M$ for each solution $\left(x_{1}(t), y_{1}(t), y_{2}(t)\right)$ of (2.1) with all $t$ large enough.

Proof Define

$$
V(t)=k_{1} x_{1}(t)+y_{1}(t)+y_{2}(t)
$$

then $t \neq n \tau, t \neq(n+l) \tau$, we have

$$
\begin{aligned}
D^{+} & V(t)+d_{1} V(t) \\
& =k_{1} x_{1}(t)\left[\left(a_{1}+d_{1}\right)-b_{1} x_{1}(t)\right]+y_{2}(t)\left[\left(a_{2}+d_{1}\right)-b_{2} y_{2}(t)\right] \\
& =-k_{1} b_{1}\left(x_{1}(t)-\frac{a_{1}+d_{1}}{2 b_{1}}\right)^{2}+\frac{k_{1}\left(a_{1}+d_{1}\right)^{2}}{4 b_{1}}-b_{2}\left(x_{2}(t)-\frac{a_{2}+d_{1}}{2 b_{2}}\right)^{2}+\frac{\left(a_{2}+\lambda\right)^{2}}{4 b_{2}} \\
& \leq \frac{k_{1}\left(a_{1}+d_{1}\right)^{2}}{4 b_{1}}+\frac{\left(a_{2}+d_{1}\right)^{2}}{4 b_{2}} \triangleq \zeta .
\end{aligned}
$$

When $t=(n+l) \tau, V\left((n+l) \tau^{+}\right)=k_{1} x_{1}\left((n+l) \tau^{+}\right)+y_{1}\left((n+l) \tau^{+}\right)+y_{2}\left((n+l) \tau^{+}\right)=k_{1} x_{1}((n+$ $l) \tau)+y_{1}((n+l) \tau)+D y_{2}((n+l) \tau)+(1-D) y_{2}((n+l) \tau)=V((n+l) \tau)$. When $t=(n+1) \tau$, $V\left((n+1) \tau^{+}\right)=k_{1} x_{1}\left((n+1) \tau^{+}\right)+y_{1}\left((n+1) \tau^{+}\right)+y_{2}\left((n+1) \tau^{+}\right)=k_{1} x_{1}((n+1) \tau)+y_{1}((n+1) \tau)+$ $\mu+y_{2}((n+1) \tau)=V((n+1) \tau)+\mu$. By Lemma 3.3, for $t \in(n \tau,(n+1) \tau]$, we have

$$
\begin{aligned}
V(t) & \leq V\left(0^{+}\right) e^{-\lambda t}+\frac{\zeta}{d_{1}}\left(1-e^{-d_{1} t}\right)+\mu \frac{e^{-d_{1}(t-\tau)}}{1-e^{-d_{1} \tau}}+\mu \frac{e^{d_{1} \tau}}{e^{d_{1} \tau}-1} \\
& \rightarrow \frac{\zeta}{d_{1}}+\mu \frac{e^{d_{1} \tau}}{e^{d_{1} \tau}-1}, \quad \text { as } t \rightarrow \infty .
\end{aligned}
$$

So $V(t)$ is uniformly ultimately bounded. Hence, by the definition of $V(t)$, we see that there exists a constant $M>0$ such that $x_{1}(t) \leq M, y_{1}(t) \leq M, y_{2}(t) \leq M$ for $t$ large enough. The proof is complete.

If $x_{1}(t)=0$, we have the following subsystem of $(2.1)$ :

$$
\left\{\begin{array}{l}
\frac{d y_{1}(t)}{d t}=-d_{1} y_{1}(t), \\
\frac{d y_{2}(t)}{d t}=y_{2}(t)\left(a_{2}-b_{2} y_{2}(t)\right),
\end{array}\right\} \quad t \neq(n+l) \tau, t \neq(n+1) \tau,
$$

We can easily obtain the analytic solution of (3.2) between pulses as follows:

$$
\left\{\begin{array}{l}
y_{1}(t)= \begin{cases}y_{1}\left(n \tau^{+}\right) e^{-d_{1}(t-n \tau)}, & t \in[n \tau,(n+l) \tau), \\
y_{1}\left((n+l) \tau^{+}\right) e^{-d_{1}(t-(n+l) \tau)}, & t \in[(n+l) \tau,(n+1) \tau),\end{cases} \\
y_{2}(t)= \begin{cases}\frac{a_{2} y_{2}\left(n \tau^{+}\right) a_{2}(t-n \tau)}{a_{2}+b_{2} y_{2}\left(n \tau^{+}\right)\left(e^{a_{2}(t-n \tau)}-1\right)}, & t \in[n \tau,(n+l) \tau), \\
\frac{a_{2} y_{2}\left((n+l) \tau^{+}\right) e^{a_{2}(t-(n+l) \tau)}}{a_{2}+b_{2} y_{2}\left((n+l) \tau^{+}\right)\left(e^{a_{2}(t-(n+l) \tau)}-1\right)}, & t \in[(n+l) \tau,(n+1) \tau) .\end{cases}
\end{array}\right.
$$


Considering the third and fourth equations of (3.2), we have

$$
\left\{\begin{array}{l}
y_{1}\left((n+l) \tau^{+}\right)=e^{-d_{1} l \tau} y_{1}\left(n \tau^{+}\right)+D \times \frac{a_{2} y_{2}\left(n \tau^{+}\right) e^{a_{2} l \tau}}{a_{2}+b_{2} y_{2}\left(n \tau^{+}\right)\left(e^{a_{2} l \tau}-1\right)}, \\
y_{2}\left((n+l) \tau^{+}\right)=(1-D) \times \frac{a_{2} y_{2}\left(n \tau^{+}\right) e^{a_{2} l \tau}}{a_{2}+b_{2} y_{2}\left(n \tau^{+}\right)\left(e^{a_{2} l \tau}-1\right)} .
\end{array}\right.
$$

Considering the fifth and sixth equations of (3.2), we also have

$$
\left\{\begin{array}{l}
y_{1}\left((n+1) \tau^{+}\right)=e^{-d_{1}(1-l) \tau} y_{1}\left((n+l) \tau^{+}\right)+\mu \\
y_{2}\left((n+1) \tau^{+}\right)=\frac{a_{2} e^{a_{2}(1-l) \tau} y_{2}\left((n+l) \tau^{+}\right)}{a_{2}+b_{2} y_{2}\left((n+l) \tau^{+}\right)\left(e^{a_{2}(1-l) \tau}-1\right)}
\end{array}\right.
$$

Substituting (3.4) into (3.5), we have the stroboscopic map of (3.2),

$$
\left\{\begin{array}{l}
y_{1}\left((n+1) \tau^{+}\right)=e^{-d_{1} \tau} y_{1}\left(n \tau^{+}\right)+\frac{D a_{2} e^{\left[a_{2} l-d_{1}(1-l)\right] \tau} y_{2}\left(n \tau^{+}\right)}{a_{2}+b_{2}\left(e^{a_{2} l \tau}-1\right) y_{2}\left(n \tau^{+}\right)}+\mu, \\
y_{2}\left((n+1) \tau^{+}\right)=\frac{(1-D) a_{2} e^{a_{2} \tau} y_{2}\left(n \tau^{+}\right)}{a_{2}+b_{2}\left(e^{a_{2} l \tau}-1\right)\left[1+(1-D) e^{a_{2} l \tau}\right] y_{2}\left(n \tau^{+}\right)}
\end{array}\right.
$$

Choosing notations $A=e^{-d_{1} \tau}<1, B_{1}=D a_{2} e^{\left[a_{2} l-d 1(1-l)\right] \tau}>0, B_{2}=(1-D) a_{2} e^{a_{2} \tau}>0, C_{1}=$ $b_{2}\left(e^{a l \tau}-1\right)>0, C_{2}=b_{2}\left(e^{a l \tau}-1\right)\left[1+(1-D) e^{a_{2} l \tau}\right]>0$, and

$$
B_{2}-a_{2}>0 \Leftrightarrow 0<D<1-e^{-a_{2} \tau}
$$

we can rewrite (3.6) as

$$
\left\{\begin{array}{l}
y_{1}\left((n+1) \tau^{+}\right)=A y_{1}\left(n \tau^{+}\right)+\frac{B_{1} y_{2}\left(n \tau^{+}\right)}{a_{2}+C_{1} y_{2}\left(n \tau^{+}\right)}+\mu \\
y_{2}\left((n+1) \tau^{+}\right)=\frac{B_{2} y_{2}\left(n \tau^{+}\right)}{a_{2}+C_{2} y_{2}\left(n \tau^{+}\right)}
\end{array}\right.
$$

Equation (3.7) has two fixed points $\left(y_{1}^{*}, 0\right)$ and $\left(y_{1}^{* *}, y_{2}^{* *}\right)$, where

$$
\begin{cases}y_{1}^{*}=\frac{\mu}{1-A}>0, & \\ y_{1}^{* *}=\frac{B_{1}\left(B_{2}-a_{2}\right)}{(1-A)\left[a_{2} C_{2}+C_{1}\left(B_{2}-a_{2}\right)\right]}+\frac{\mu}{1-A}>0, & 0<D<1-e^{-a_{2} \tau}, \\ y_{2}^{* *}=\frac{B_{2}-a_{2}}{C_{2}}>0, & 0<D<1-e^{-a_{2} \tau} .\end{cases}
$$

\section{Lemma 3.5}

(i) If $1-e^{-a_{2} \tau}<D<1$ holds, the fixed point $\left(y_{1}^{*}, 0\right)$ of (3.6) or (3.7) is globally asymptotically stable.

(ii) If $0<D<1-e^{-a_{2} \tau}$ holds, the fixed point $\left(y_{1}^{* *}, y_{2}^{* *}\right)$ of (3.6) or (3.7) is globally asymptotically stable.

Proof For convenience, we take the notation $\left(y_{1}^{n}, y_{2}^{n}\right)=\left(y_{1}\left(n \tau^{+}\right), y_{2}\left(n \tau^{+}\right)\right)$. The linear form of (3.5) can be written as

$$
\left(\begin{array}{l}
y_{1}^{n+1} \\
y_{2}^{n+1}
\end{array}\right)=M\left(\begin{array}{l}
y_{1}^{n} \\
y_{2}^{n}
\end{array}\right)
$$

Obviously, the near dynamics of $\left(y_{1}^{*}, 0\right)$ and $\left(y_{1}^{* *}, y_{2}^{* *}\right)$ are determined by the linear System (3.6) or (3.7). The stabilities of $\left(y_{1}^{*}, 0\right)$ and $\left(y_{1}^{* *}, y_{2}^{* *}\right)$ are determined by the eigenvalue of 
$M$ less than 1. If $M$ satisfies the Jury criterion [34], we can know the eigenvalue of $M$ less than 1 ,

$$
1-\operatorname{tr} M+\operatorname{det} M>0
$$

(i) If $1-e^{-a_{2} \tau}<D<1$ holds, that is, $\frac{B_{2}}{a_{2}}<1$, we can easily see that $\left(y_{1}^{*}, 0\right)$ is a unique fixed point of (3.6) or (3.7), and

$$
M=\left(\begin{array}{cc}
A & \frac{B_{1}}{a_{2}} \\
0 & \frac{B_{2}}{a_{2}}
\end{array}\right) .
$$

For

$$
\begin{aligned}
1-\operatorname{tr} M+\operatorname{det} M & =1-\left(A+\frac{B_{2}}{a_{2}}\right)+\left(A \times \frac{B_{2}}{a_{2}}\right) \\
& =(1-A)\left(1-\frac{B_{2}}{a_{2}}\right)>0 .
\end{aligned}
$$

From the Jury criterion, $\left(y_{1}^{*}, 0\right)$ is locally stable, then it is globally asymptotically stable.

(ii) If $0<D<1-e^{-a_{2} \tau}$ holds, that is, $\frac{a_{2}}{B_{2}}<1$, we can easily see that $\left(y_{1}^{* *}, y_{2}^{* *}\right)$ is a unique fixed point of (3.6) or (3.7), and

$$
M=\left(\begin{array}{cc}
A & \frac{B_{1} a_{2}}{\left(a_{2}+C_{1} v_{2}^{* *}\right)^{2}} \\
0 & \frac{B_{2} a_{2}}{\left(a_{2}+C_{2} y_{2}^{* *}\right)^{2}}
\end{array}\right) .
$$

For

$$
\begin{aligned}
1-\operatorname{tr} M+\operatorname{det} M & =1-\left(A+\frac{B_{2} a_{2}}{\left(a_{2}+C_{2} y_{2}^{* *}\right)^{2}}\right)+\left(A \times \frac{B_{2} a_{2}}{\left(a_{2}+C_{2} y_{2}^{* *}\right)^{2}}\right) \\
& =(1-A)\left[1-\frac{B_{2} a_{2}}{\left(a_{2}+C_{2} y_{2}^{* *}\right)^{2}}\right] \\
& =(1-A)\left(1-\frac{a_{2}}{B_{2}}\right)>0 .
\end{aligned}
$$

From the Jury criterion, $\left(y_{1}^{* *}, y_{2}^{* *}\right)$ is locally stable, then it is globally asymptotically stable. This completes the proof.

\section{Lemma 3.6}

(i) If $0<D<1-e^{-a_{2} \tau}$ holds, the periodic solution $\left(\widetilde{y_{1}(t)}, \widetilde{y_{2}(t)}\right)$ of System (3.2) is globally asymptotically stable, where

$$
\left\{\begin{array}{l}
\widetilde{y_{1}(t)}= \begin{cases}y_{1}^{* *} e^{-d_{1}(t-n \tau)}, & t \in[n \tau,(n+l) \tau), \\
y_{1}^{* * *} e^{-d_{1}(t-(n+l) \tau)}, & t \in[(n+l) \tau,(n+1) \tau),\end{cases} \\
\widetilde{y_{2}(t)}= \begin{cases}\frac{a_{2} y_{2}^{* *} e^{a_{2}(t-n \tau)}}{a_{2}+b_{2} y_{2}^{* *}\left(e^{a_{2}(t-n \tau)}-1\right)}, & t \in[n \tau,(n+l) \tau), \\
\frac{a_{2} y_{2}^{* * *} a_{2}(t-(n+l) \tau)}{a_{2}+b_{2} y_{2}^{* * *}\left(e^{a_{2}(t-(n+l) \tau)}-1\right)}, & t \in[(n+l) \tau,(n+1) \tau),\end{cases}
\end{array}\right.
$$


where $y_{1}^{* *}$ and $y_{2}^{* *}$ are determined as (3.8), $y_{1}^{* * *}$ and $y_{2}^{* * *}$ are defined as

$$
\left\{\begin{array}{l}
y_{1}^{* * *}=e^{-d_{1} l \tau} y_{1}^{* *}+D \times \frac{a_{2} y_{2}^{* *} e^{a_{2} l \tau}}{a_{2}+b_{2} y_{2}^{* *}\left(e^{a_{2} l \tau}-1\right)}, \\
y_{2}^{* * *}=(1-D) \times \frac{a_{2} y_{2}^{* *} e^{a_{2} l \tau}}{a_{2}+b_{2} y_{2}^{* *}\left(e^{a_{2} l \tau}-1\right)} .
\end{array}\right.
$$

(ii) If $1-e^{-a_{2} \tau}<D<1$ holds, the periodic solution $\left(\widehat{y_{1}(t)}, 0\right)$ of System (3.2) is globally asymptotically stable, where

$$
\widehat{y_{1}(t)}= \begin{cases}y_{1}^{*} e^{-d_{1}(t-n \tau)}, & t \in[n \tau,(n+l) \tau), \\ y_{1}^{* * * *} e^{-d_{1}(t-(n+l) \tau)}, & t \in[(n+l) \tau,(n+1) \tau),\end{cases}
$$

where $y_{1}^{* * * *}=e^{-d_{1} \tau} y_{1}^{*}$, and $y_{1}^{*}$ is determined as (3.8).

Therefore, if $y_{1}(0)=0.5, y_{2}(0)=0.5, d_{1}=0.6, a_{2}=0.1, b_{2}=0.2, \mu=0.4, l=0.25, \tau=1$, $D=0.15$, then $0.15=D>D^{*}=1-e^{-a_{2} \tau} \doteq 0.0952$; the predator population $y_{2}(t)$ will go into extinction. Its dynamical behaviors can be seen in Figure 1 . If $y_{1}(0)=0.5, y_{2}(0)=0.5$, $d_{1}=0.6, a_{2}=0.1, b_{2}=0.2, \mu=0.4, l=0.25, \tau=1, D=0.05$, then $0.05=D<D^{*} \doteq 0.0952$; the predator population are permanent. Its dynamical behaviors can be seen in Figure 2 . Obviously, the diffusion rate between the patches affects the dynamics of System (3.2).

(a)

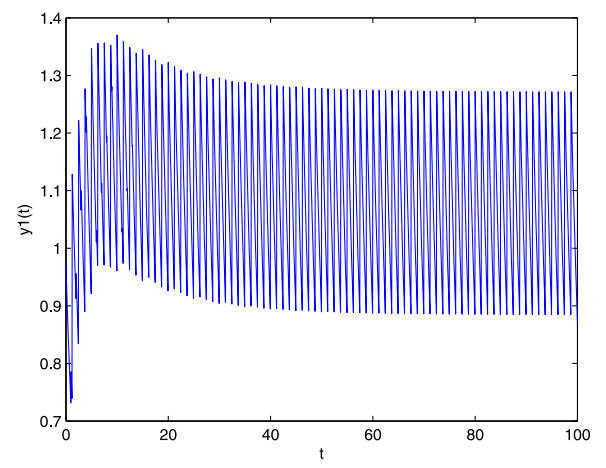

(b)

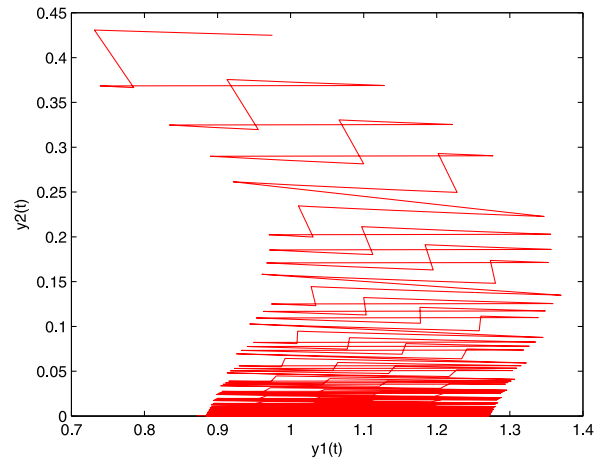

(c)

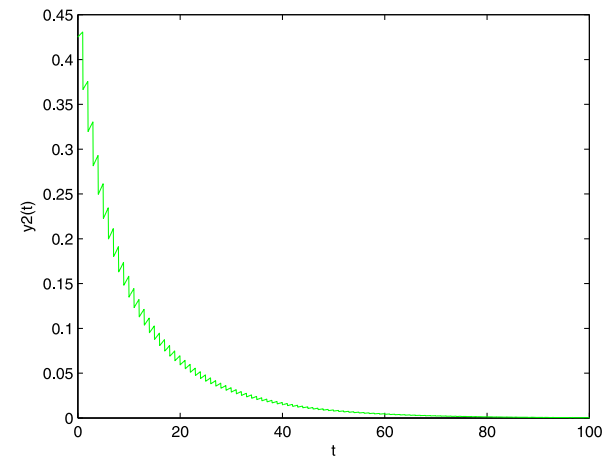

Figure 1 Globally asymptotically stable $y_{2}(t)$ extinction periodic solution with $y_{1}(0)=0.5, y_{2}(0)=0.5$, $d_{1}=0.6, a_{2}=0.1, b_{2}=0.2, \mu=0.4, I=0.25, \boldsymbol{\tau}=1, D=0.15$. (a) Time series of $y_{1}(t)$; (b) time series of $y_{2}(t)$; (c) the phase portrait of a globally asymptotically stable periodic solution $\left(\widehat{y_{1}(t)}, 0\right)$ of System (3.2). 
(a)

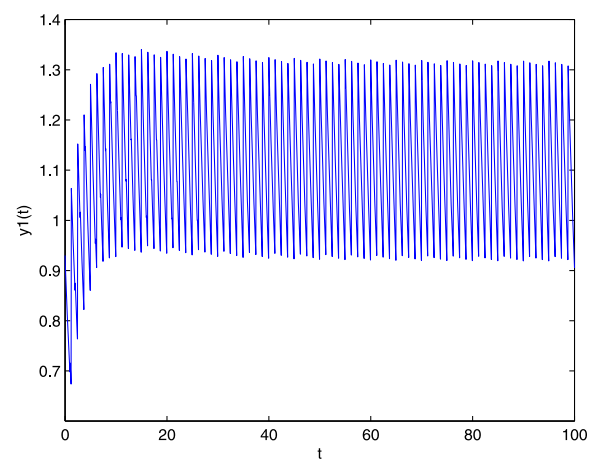

(b)

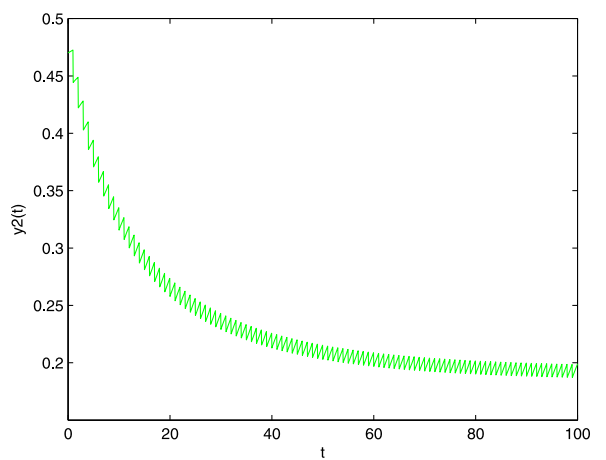

(c)

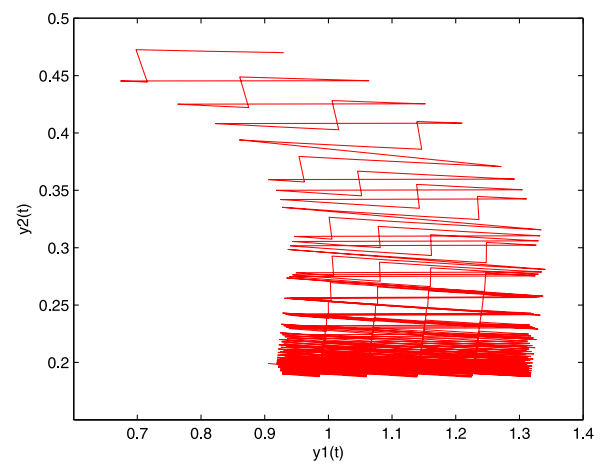

Figure 2 Permanence for System (3.2) with $y_{1}(0)=0.5, y_{2}(0)=0.5, d_{1}=0.6, a_{2}=0.1, b_{2}=0.2, \mu=0.4$, $I=0.25, \tau=1, D=0.05$. (a) Time series of $y_{1}(t)$; (b) time series of $y_{2}(t)$; (c) the phase portrait of permanence for System (3.2).

\section{The dynamics}

Theorem 4.1 If

$$
1-e^{-a_{2} \tau}<D<1
$$

and

$$
a_{1} \tau<\frac{\beta_{1}}{d_{1}}\left[y_{1}^{*}\left(1-e^{-d_{1} l \tau}\right)+y_{1}^{* * * *}\left(e^{-d_{1} l \tau}-e^{-d_{1} \tau}\right)\right]
$$

hold, the prey-extinction boundary periodic solution $\left(0, \widehat{y_{1}(t)}, 0\right)$ of $(2.1)$ is globally asymptotically stable, where $y_{1}^{*}$ and $y_{1}^{* * * *}$ are defined as (3.8) and (3.15).

Proof First, we prove the local stability of the prey-extinction boundary periodic solution $\left(0, \widehat{y_{1}(t)}, 0\right)$ of $(2.1)$. Defining $x_{1}(t)=x_{1}(t), y_{11}(t)=y_{1}(t)-\widehat{y_{1}(t)}, y_{2}(t)=y_{2}(t)$, then we have the following linearly similar system for $(2.1)$ which concerns one periodic solution $\left(0, \widehat{y_{1}(t)}, 0\right)$ :

$$
\left(\begin{array}{c}
\frac{d x_{1}(t)}{d t} \\
\frac{d y_{11}(t)}{d t} \\
\frac{d y_{2}(t)}{d t}
\end{array}\right)=\left(\begin{array}{ccc}
a_{1}-\beta_{1} \widehat{y_{1}(t)} & 0 & 0 \\
k_{1} \beta_{1} \widehat{y_{1}(t)} & -d_{1} & 0 \\
0 & 0 & a_{2}
\end{array}\right)\left(\begin{array}{l}
x_{1}(t) \\
y_{11}(t) \\
y_{2}(t)
\end{array}\right) .
$$


It is easy to obtain the fundamental solution matrix

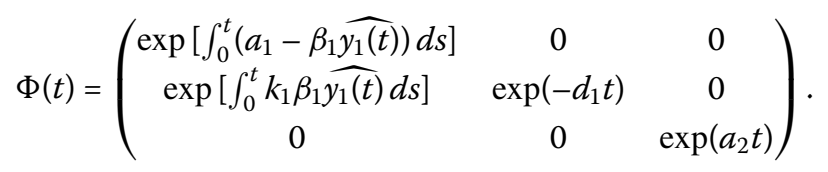

The linearization of the fourth, fifth, and sixth equations of (2.1) is

$$
\left(\begin{array}{l}
x_{1}\left((n+l) \tau^{+}\right) \\
y_{11}\left((n+l) \tau^{+}\right) \\
y_{2}\left((n+l) \tau^{+}\right)
\end{array}\right)=\left(\begin{array}{ccc}
1 & 0 & 0 \\
0 & 1 & D \\
0 & 0 & 1-D
\end{array}\right)\left(\begin{array}{l}
x_{1}((n+l) \tau) \\
y_{11}((n+l) \tau) \\
y_{2}((n+l) \tau)
\end{array}\right) \text {. }
$$

The linearization of the seventh, eighth, and ninth equations of (2.1) is

$$
\left(\begin{array}{l}
x_{1}\left((n+1) \tau^{+}\right) \\
y_{11}\left((n+1) \tau^{+}\right) \\
y_{2}\left((n+1) \tau^{+}\right)
\end{array}\right)=\left(\begin{array}{lll}
1 & 0 & 0 \\
0 & 1 & 0 \\
0 & 0 & 1
\end{array}\right)\left(\begin{array}{l}
x_{1}((n+1) \tau) \\
y_{11}((n+1) \tau) \\
y_{2}((n+1) \tau)
\end{array}\right) \text {. }
$$

The stability of the periodic solution $\left(0, \widehat{y_{1}(t)}, 0\right)$ is determined by the eigenvalues of

$$
M=\left(\begin{array}{ccc}
1 & 0 & 0 \\
0 & 1 & D \\
0 & 0 & 1-D
\end{array}\right)\left(\begin{array}{ccc}
1 & 0 & 0 \\
0 & 1 & 0 \\
0 & 0 & 1
\end{array}\right) \Phi(\tau)
$$

where

$$
\begin{aligned}
& \lambda_{1}=\exp \left[\int_{0}^{\tau}\left(a_{1}-\beta_{1} \widehat{y_{1}(t)}\right) d s\right], \\
& \lambda_{2}=e^{-d_{1} \tau}<1,
\end{aligned}
$$

and

$$
\lambda_{3}=(1-D) e^{a_{2} \tau} .
$$

According to condition (4.1), (4.2), and the Floquet theory [21], i.e.

$$
\exp \left[\int_{0}^{\tau}\left(a_{1}-\beta_{1} \widehat{y_{1}(s)}\right) d s\right]<1
$$

and

$$
(1-D) e^{a_{2} \tau}<1,
$$

we have

$$
\lambda_{1}<1
$$


and

$\lambda_{3}<1$,

the prey-extinction boundary periodic solution $\left(0, \widehat{y_{1}(t)}, 0\right)$ of $(2.1)$ is locally stable.

In the following, we will prove the global attraction. From condition (4.2), we can choose an $\varepsilon>0$ such that

$$
\rho=\exp \left[\int_{0}^{\tau}\left(a_{1}-\beta_{1}\left(\widehat{y_{1}(s)}-\varepsilon\right)\right) d s\right]<1 .
$$

From the second equation of (2.1), we notice that $\frac{d y_{1}(t)}{d t} \geq-d_{1} y_{1}(t)$. Then we consider the following impulsive comparative differential equation:

$$
\left\{\begin{array}{l}
\frac{d y_{21}(t)}{d t}=-d_{1} y_{21}(t), \\
\frac{d y_{22}(t)}{d t}=y_{22}(t)\left(a_{2}-b_{2} y_{22}(t)\right),
\end{array}\right\} \quad t \neq(n+l) \tau, t \neq(n+1) \tau,
$$

From Lemma 3.6 and the comparison theorem of impulsive equations (see Theorem 3.1.1 in [29]), we have $y_{1}(t) \geq y_{21}(t), y_{2}(t) \geq y_{22}(t)$, and $y_{21}(t) \rightarrow \widehat{y_{1}(s)}, y_{22}(t) \rightarrow 0$, as $t \rightarrow \infty$. Then

$$
\left\{\begin{array}{l}
y_{1}(t) \geq y_{21}(t) \geq \widehat{y_{1}(s)}-\varepsilon \\
y_{2}(t) \geq y_{22}(t) \geq-\varepsilon
\end{array}\right.
$$

for all $t$ large enough. For convenience, we may assume (4.4) holds for all $t \geq 0$. From (2.1) and (4.4), we get

$$
\frac{d x_{1}(t)}{d t} \leq\left[a_{1}-\beta_{1}\left(\widehat{y_{1}(s)}-\varepsilon\right)\right] x_{1}(t)
$$

So $x_{1}((n+1) \tau) \leq x_{1}\left(n \tau^{+}\right) \exp \left[\int_{n \tau}^{(n+1) \tau}\left(a_{1}-\beta_{1}\left(\widehat{y_{1}(s)}-\varepsilon\right)\right) d s\right]$. Hence, $x_{1}(n \tau) \leq x_{1}\left(0^{+}\right) \rho^{n}$ and $x_{1}(n \tau) \rightarrow 0$ as $n \rightarrow \infty$, therefore $x_{1}(t) \rightarrow 0(i=1,2)$ as $t \rightarrow \infty$.

Next, we will prove that $y_{1}(t) \rightarrow \widehat{y_{1}(t)}$ and $y_{2}(t) \rightarrow 0$ as $t \rightarrow \infty$. For $0<\varepsilon_{1}<\frac{d_{1}}{k_{1} \beta_{1}}$ small enough, there must exist a $t_{0}>0$ such that $0<x_{1}(t)<\varepsilon_{1}$ for all $t \geq t_{0}$. Without loss of generality, we may assume that $0<x_{1}(t)<\varepsilon_{1}$ for all $t \geq 0$. For System (2.1) we have

$$
-d_{1} y_{1}(t) \leq \frac{d y_{1}(t)}{d t} \leq-\left(d_{1}-k_{1} \beta_{1} \varepsilon_{1}\right) y_{1}(t),
$$

then we have $y_{21}(t) \leq y_{1}(t) \leq y_{31}(t), y_{22}(t) \leq y_{2}(t) \leq y_{32}(t)$, and $y_{21}(t) \rightarrow \widehat{y_{1}(t)}, \widehat{y_{22}(t)} \rightarrow 0$, $y_{31}(t) \rightarrow \widehat{y_{31}(t)}, \widehat{y_{32}(t)} \rightarrow 0$, as $t \rightarrow \infty$. Meanwhile $\left(y_{21}(t), y_{22}(t)\right)$ and $\left(y_{31}(t), y_{32}(t)\right)$ are the 
solutions of (4.3) and

$$
\left\{\begin{array}{l}
\frac{d y_{31}(t)}{d t}=-\left(d_{1}-k_{1} \beta_{1} \varepsilon_{1}\right) y_{31}(t), \\
\left.\begin{array}{l}
\frac{d y_{32}(t)}{d t}=y_{32}(t)\left(a_{2}-b_{2} y_{32}(t)\right), \\
\triangle y_{31}(t)=D y_{32}(t), \\
\triangle y_{32}(t)=-D y_{32}(t),
\end{array}\right\} \quad t=(n+l) \tau \\
\left.\begin{array}{l}
\triangle y_{31}(t)=\mu, \\
\triangle y_{32}(t)=0,
\end{array}\right\} \quad t=(n+1) \tau,
\end{array}\right.
$$

respectively. We have

$$
\widehat{y_{31}(t)}= \begin{cases}y_{31}^{*} e^{-\left(d_{1}-k_{1} \beta_{1} \varepsilon_{1}\right)(t-n \tau)}, & t \in[n \tau,(n+l) \tau), \\ y_{31}^{* * * * *} e^{-\left(d_{1}-k_{1} \beta_{1} \varepsilon_{1}\right)(t-(n+l) \tau)}, & t \in[(n+l) \tau,(n+1) \tau),\end{cases}
$$

where $y_{31}^{* * *}=e^{-\left(d_{1}-k_{1} \beta_{1} \varepsilon_{1}\right) \tau} y_{31}^{*}$, and $y_{31}^{*}$ is determined as

$$
y_{31}^{*}=\frac{\mu}{1-e^{-\left(d_{1}-k_{1} \beta_{1} \varepsilon_{1}\right) \tau}} .
$$

For any $\varepsilon_{2}>0$, there exists a $t_{1}, t>t_{1}$ such that

$$
\widehat{y_{21}(t)}-\varepsilon_{2}<y_{1}(t)<\widehat{y_{31}(t)}+\varepsilon_{2}
$$

and

$$
\widehat{y_{22}(t)}-\varepsilon_{2}<y_{2}(t)<\widehat{y_{32}(t)}+\varepsilon_{2}
$$

Let $\varepsilon_{1} \rightarrow 0$, so we have

$$
\widehat{y_{1}(t)}-\varepsilon_{2}<y_{1}(t)<\widehat{y_{1}(t)}+\varepsilon_{2}
$$

and

$$
-\varepsilon_{2}<y_{2}(t)<+\varepsilon_{2},
$$

for $t$ large enough, which implies $y_{1}(t) \rightarrow \widehat{y_{1}(t)}$ and $y_{2}(t) \rightarrow 0$ as $t \rightarrow \infty$. This completes the proof.

We can easily prove Theorem 4.2 similar to Theorem 4.1.

\section{Theorem 4.2 If}

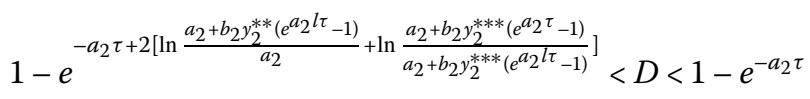

and

$$
a_{1} \tau<\frac{\beta_{1}}{d_{1}}\left[y_{1}^{* *}\left(1-e^{-d_{1} l \tau}\right)+y_{1}^{* * *}\left(e^{-d_{1} l \tau}-e^{-d_{1} \tau}\right)\right]
$$


hold, the prey-extinction boundary periodic solution $\left(0, \widetilde{y_{1}(t)}, \widetilde{y_{2}(t)}\right)$ of $(2.1)$ is globally asymptotically stable, where $y_{1}^{* *}, y_{1}^{* * *}, y_{2}^{* *}$, and $y_{2}^{* * *}$ are defined as (3.8) and (3.14).

The next task is to investigate the permanence of System (2.1).

Definition 4.3 System (2.1) is said to be permanent if there are constants $m, M>0$ (independent of the initial value) and a finite time $T_{0}$ such that for all solutions $\left(x_{1}(t), y_{1}(t), y_{2}(t)\right)$ with all initial values $x_{1}\left(0^{+}\right)>0, y_{1}\left(0^{+}\right)>0, y_{2}\left(0^{+}\right)>0, m \leq x_{1}(t) \leq M, m \leq y_{1}(t) \leq M$, $m \leq y_{2}(t) \leq M$ hold for all $t \geq T_{0}$. Here $T_{0}$ may depend on the initial values $\left(x_{1}\left(0^{+}\right), y_{1}\left(0^{+}\right), y_{2}\left(0^{+}\right)\right)$.

\section{Theorem 4.4 If}

$$
0<D<1-e^{-a_{2} \tau}
$$

and

$$
a_{1} \tau>\frac{\beta_{1}}{d_{1}}\left[y_{1}^{* *}\left(1-e^{-d_{1} l \tau}\right)+y_{1}^{* * *}\left(e^{-d_{1} l \tau}-e^{-d_{1} \tau}\right)\right]
$$

hold, System (2.1) is permanent, where $y_{1}^{* *}$ and $y_{1}^{* * *}$ are defined as (3.8) and (3.14).

Proof Suppose $\left(x_{1}(t), y_{1}(t), y_{2}(t)\right)$ is a solution of $(2.1)$ with $x_{1}(0)>0, y_{1}(0)>0, y_{2}(0)>0$. By Lemma 3.4, we have proved there exists a constant $M>0$ such that $x_{1}(t) \leq M, y_{1}(t) \leq M$, $y_{2}(t) \leq M$, for $t$ large enough. From (2.1), Theorem 4.1, and condition (4.11), we have

$$
\left\{\begin{aligned}
y_{1}(t) & >\widetilde{y_{1}(t)}-\varepsilon_{2} \\
& >y_{1}^{* *} e^{-d_{1} l \tau}+y_{1}^{* * *} e^{-d_{1}(1-l) \tau}-\varepsilon_{2} \triangleq m_{1}, \\
y_{2}(t) & >\widetilde{y_{2}(t)}-\varepsilon_{2} \\
& >\frac{a_{2} y_{1}^{* *} e^{a_{2} l \tau}}{a_{2}+b_{2} y_{1}^{* *}\left(e^{a_{2} l \tau}-1\right)}+\frac{a_{2} y_{1 * *}^{* *} e^{a_{2}(1-l) \tau}}{a_{2}+b_{2} y_{1}^{* * *}\left(e^{a_{2}(1-l) \tau}-1\right)}-\varepsilon_{2} \triangleq m_{2},
\end{aligned}\right.
$$

for $\varepsilon_{2}$ small enough. So we only need to find $m_{3}>0$ and $\varepsilon_{3}$ such that $x_{1}(t)>m_{3}$ for $t$ large enough. Otherwise, we can select $m_{4}>0$ small enough satisfying $m_{4}<\frac{d_{1}}{k_{1} \beta_{1}}$, and we prove $x_{1}(t)<m_{4}$ cannot hold for $t \geq 0$. By condition (4.12) and choosing $\varepsilon_{3}$ small enough, we can obtain

$$
\begin{aligned}
\sigma_{i}= & a_{1} \tau \\
& -\frac{\beta_{1}}{\left(d_{1}-k_{1} \beta_{1} m_{4}\right)}\left[y_{41}^{* *}\left(1-e^{-\left(d_{1}-k_{1} \beta_{1} m_{4}\right) l \tau}\right)+y_{41}^{* * *}\left(e^{-\left(d_{1}-k_{1} \beta_{1} m_{4}\right) l \tau}-e^{-\left(d_{1}-k_{1} \beta_{1} m_{4}\right) \tau}\right)\right] \\
& -\beta_{1} \varepsilon_{3} \tau
\end{aligned}
$$


(a)

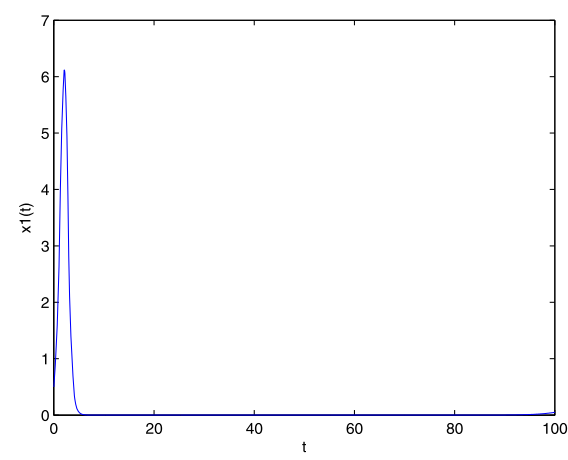

(c)

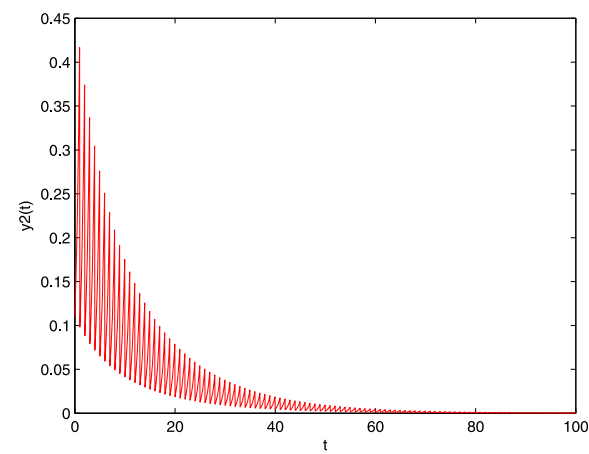

(b)

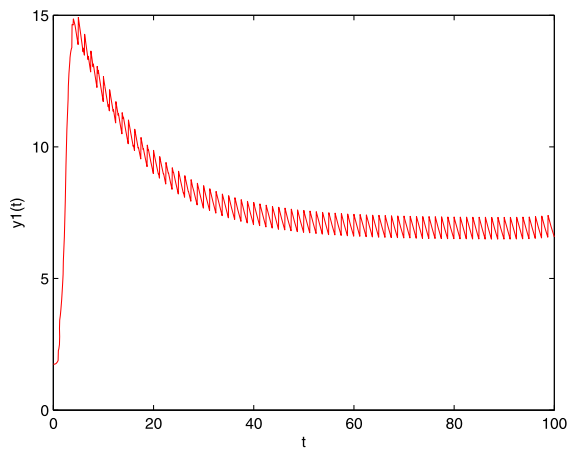

(d)

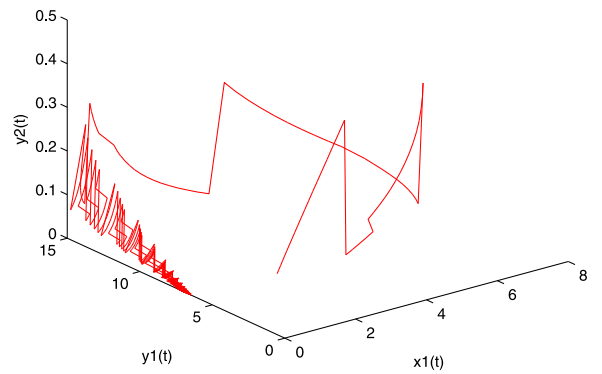

Figure 3 Globally asymptotically stable prey-extinction periodic solution $\left(0, \widehat{y_{1}(t)}, 0\right)$ of System $(2.1)$ with $x_{1}(0)=0.5, y_{1}(0)=0.5, y_{2}(0)=0.5, a_{1}=2.4, b_{1}=0.1, a_{2}=1.5, b_{2}=0.21, \beta_{1}=0.3, k_{1}=0.5$,

$\boldsymbol{\mu}=\mathbf{0 . 8 6}, \boldsymbol{d}_{\mathbf{1}}=\mathbf{0 . 1}, \boldsymbol{\tau}=\mathbf{1}, \boldsymbol{I}=\mathbf{0 . 2 5}, \boldsymbol{D}=\mathbf{0 . 7 8}$. (a) Time series of $x_{1}(t)$; (b) time series of $y_{1}(t)$; (c) time-series of $y_{2}(t) ;(\mathbf{d})$ the phase portrait of globally asymptotically stable periodic solution $\left(0, \widehat{y_{1}(t)}, 0\right)$ of System $(2.1)$.

with $y_{41}^{* *}$ and $y_{41}^{* * *}$ defined as (4.17) and (4.18). Then

$$
\left\{\begin{array}{l}
\left.\begin{array}{l}
\frac{d y_{1}(t)}{d t}<-\left(d_{1}-k_{1} \beta_{1} m_{4}\right) y_{1}(t), \\
\frac{d y_{2}(t)}{d t}=y_{2}(t)\left(a_{2}-b_{2} y_{2}(t)\right),
\end{array}\right\} \quad t \neq(n+l) \tau, t \neq(n+1) \tau, \\
\triangle y_{1}(t)=D y_{2}(t), \\
\triangle y_{2}(t)=-D y_{2}(t), \\
\triangle y_{1}(t)=\mu, \\
\triangle y_{2}(t)=0,
\end{array}\right\} \quad t=(n+l) \tau,
$$

By Lemma 3.6, we have $y_{1}(t) \leq y_{41}(t), y_{2}(t) \leq y_{42}(t)$ and $y_{41}(t) \rightarrow \overline{y_{41}(t)}, y_{42}(t) \rightarrow \overline{y_{42}(t)}$, $t \rightarrow \infty$, where $\left(y_{41}(t), y_{42}(t)\right)$ is the solution of

$$
\left\{\begin{array}{l}
\begin{array}{l}
\frac{d y_{41}(t)}{d t}=-\left(d_{1}-k_{1} \beta_{1} m_{4}\right) y_{41}(t), \\
\frac{d y_{42}(t)}{d t}=y_{42}(t)\left(a_{2}-b_{2} y_{42}(t)\right),
\end{array} \\
\triangle y_{41}(t)=D y_{42}(t), \\
\triangle y_{42}(t)=-D y_{42}(t), \\
\triangle y_{41}(t)=\mu, \\
\triangle y_{42}(t)=0,
\end{array}\right\} \quad t=(n+l) \tau, \quad t=(n+1) \tau, t \neq(n+1) \tau,
$$


(a)

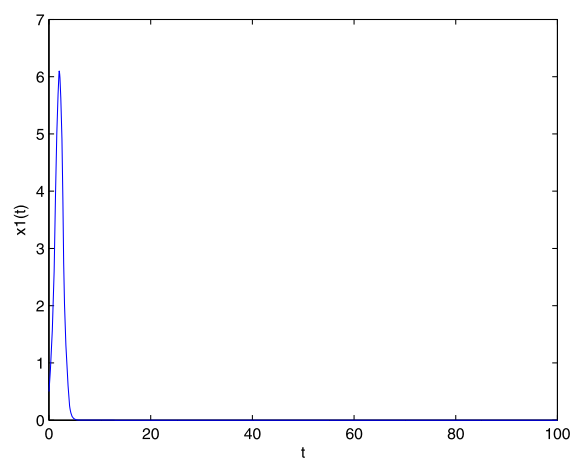

(c)

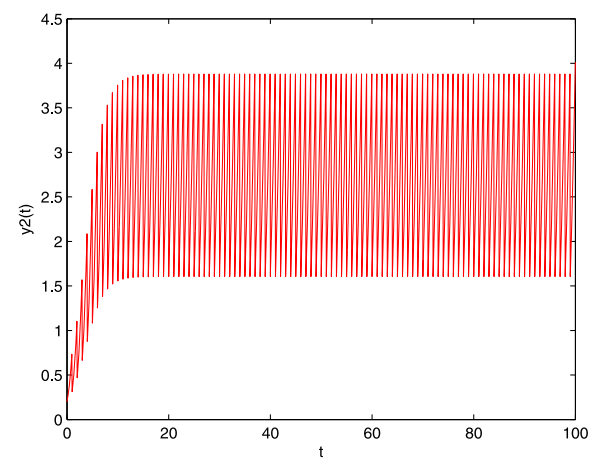

(b)

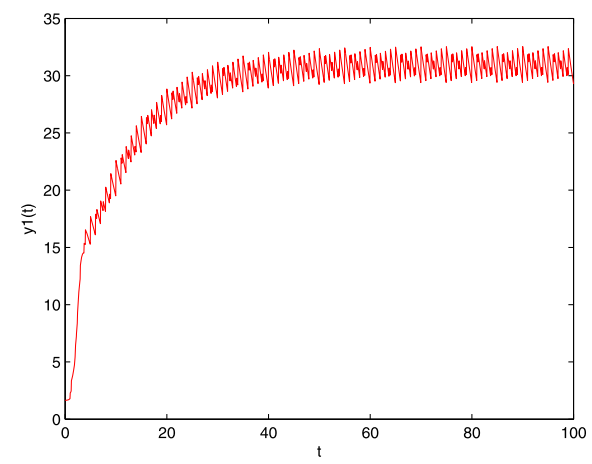

(d)

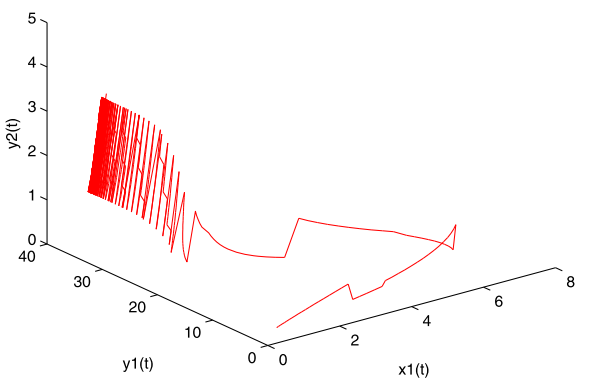

Figure 4 Globally asymptotically stable prey-extinction periodic solution $\left(0, \widetilde{y_{1}(t)}, \widetilde{y_{2}(t)}\right)$ of System (2.1) with $x_{1}(0)=0.5, y_{1}(0)=0.5, y_{2}(0)=0.5, a_{1}=2.4, b_{1}=0.1, a_{2}=1.5, b_{2}=0.21, \beta_{1}=0.3$, $k_{1}=0.5, \boldsymbol{\mu}=0.86, \boldsymbol{d}_{1}=0.1, \boldsymbol{\tau}=1, I=0.25, D=0.6$. (a) Time-series of $x_{1}(t)$; (b) time-series of $y_{1}(t)$;

(c) time-series of $y_{2}(t) ;(\mathbf{d})$ the phase portrait of globally asymptotically stable periodic solution $\left(0, \widetilde{y_{1}(t)}, \widetilde{\left.y_{2}(t)\right)}\right.$ of System (2.1).

with

$$
\left\{\begin{array}{l}
\widetilde{y_{41}(t)}= \begin{cases}y_{41}^{* *} e^{-\left(d_{1}-k_{1} \beta_{1} m_{4}\right)(t-n \tau)}, & t \in[n \tau,(n+l) \tau), \\
y_{41}^{* *} e^{-\left(d_{1}-k_{1} \beta_{1} m_{4}\right)(t-(n+l) \tau)}, & t \in[(n+l) \tau,(n+1) \tau),\end{cases} \\
\widetilde{y_{42}(t)}= \begin{cases}\frac{a_{2} y_{42}^{* *} e_{2}(t-n \tau)}{\left.a_{2}+b_{2} y_{42}^{* *}\left(e^{a_{2}(t-n \tau)}\right)-1\right)}, & t \in[n \tau,(n+l) \tau), \\
\frac{a_{2} y_{42}^{* *} e^{a_{2}(t-(n+l) \tau)}}{a_{2}+b_{2} y_{42}^{* *}\left(e^{a_{2}(t-(n+l) \tau)}-1\right)}, & t \in[(n+l) \tau,(n+1) \tau),\end{cases}
\end{array}\right.
$$

where $y_{41}^{* *}$ and $y_{42}^{* *}$ are determined as

$$
\begin{cases}y_{41}^{* *}=\frac{B_{41}\left(B_{42}-a_{2}\right)}{\left(1-A_{4}\right)\left[a_{2} C_{42}+C_{41}\left(B_{42}-a_{2}\right)\right]}+\frac{\mu}{1-A_{4}}>0, & 0<D<1-e^{-a_{2} \tau}, \\ y_{42}^{* *}=\frac{B_{42}-a_{2}}{C_{42}}>0, & 0<D<1-e^{-a_{2} \tau},\end{cases}
$$

with $A_{4}=e^{-\left(d_{1}-k_{1} \beta_{1} m_{4}\right) \tau}<1, B_{41}=D a_{2} e^{a_{2} l \tau}>0, B_{42}=(1-D) a_{2} e^{a_{2} \tau}>0, C_{41}=b_{2}\left(e^{a l \tau}-1\right)>0$, $C_{42}=b_{2}\left(e^{a l \tau}-1\right)\left[1+(1-D) e^{a_{2} l \tau}\right]>0$, and $y_{1}^{* * *}$ and $y_{2}^{* * *}$ are defined as

$$
\left\{\begin{array}{l}
y_{41}^{* * *}=e^{-\left(d_{1}-k_{1} \beta_{1} m_{4}\right) l \tau} y_{41}^{* *}+D \times \frac{a_{2} y_{42}^{* *} a_{2} l \tau}{a_{2}+b_{2} y_{42}^{* *}\left(e^{a_{2} l \tau}-1\right)}, \\
y_{42}^{* * *}=(1-D) \times \frac{a_{2} y_{42}^{* *} a_{2} a_{2} l \tau}{a_{2}+b_{2} y_{42}^{* *}\left(e^{a_{2} l \tau}-1\right)} .
\end{array}\right.
$$


(a)

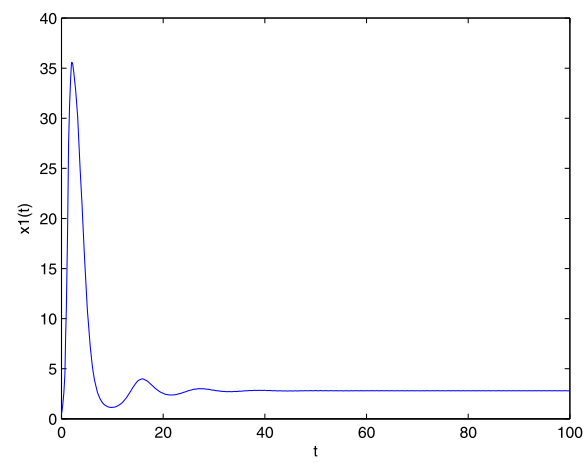

(c)

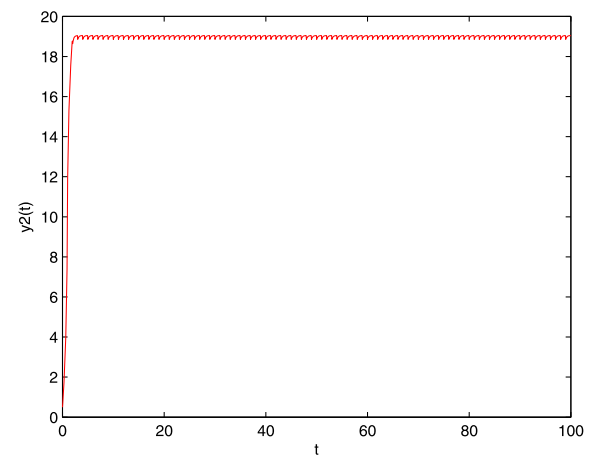

(b)

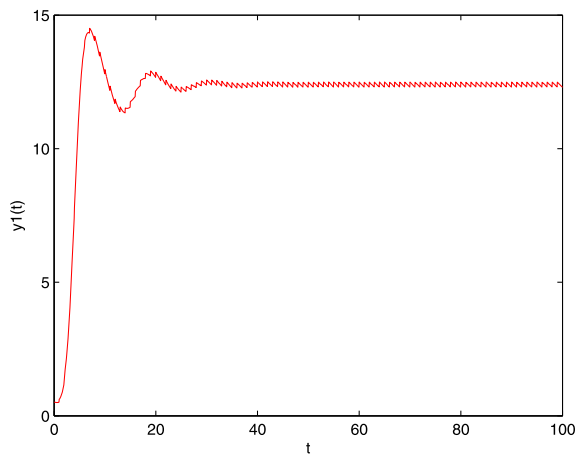

(d)

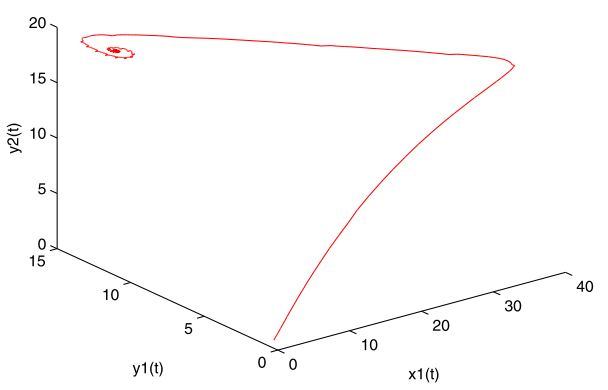

Figure 5 Permanence for System (2.1) with $x_{1}(0)=0.5, y_{1}(0)=0.5, y_{2}(0)=0.5, a_{1}=4, b_{1}=0.1, a_{2}=4$, $b_{2}=0.21, \beta_{1}=0.3, k_{1}=0.1, \mu=0.01, d_{1}=0.1, \tau=1, I=0.25, D=0.01$. (a) Time-series of $x_{1}(t)$; (b) time-series of $y_{1}(t) ;(\mathbf{c})$ time-series of $y_{2}(t)$; (d) the phase portrait of permanence for System (2.1),

Therefore, there exist $T_{1}>0$ and $\varepsilon_{3}>0$ such that

$$
y_{1}(t) \leq y_{41}(t) \leq \overline{y_{41}(t)}-\varepsilon_{3}
$$

and

$$
y_{2}(t) \leq y_{42}(t) \leq \overline{y_{42}(t)}-\varepsilon_{3} .
$$

Then

$$
\frac{d x_{1}(t)}{d t} \geq\left[a_{1}-\beta_{1}\left(\overline{y_{1}(t)}-\varepsilon_{3}\right)\right] x_{1}(t)
$$

for $t \geq T_{1}$, let $N_{1} \in N$ and $N_{1} \tau>T_{1}$. Integrating (4.19) on $(n \tau,(n+1) \tau), n \geq N_{1}$, we have

$$
x_{1}((n+1) \tau) \geq x_{1}\left(n \tau^{+}\right) \exp \left(\int_{n \tau}^{(n+1) \tau}\left[a_{1}-\beta_{1}\left(\overline{y_{1}(t)}-\varepsilon_{3}\right)\right] d t\right)=x_{1}(n \tau) e^{\sigma},
$$

then $x_{1}\left(\left(N_{1}+k\right) \tau\right) \geq x_{1}\left(N_{1} \tau^{+}\right) e^{k \sigma} \rightarrow \infty$, as $k \rightarrow \infty$, which is a contradiction to the boundedness of $x_{1}(t)$. Hence, there exists a $t_{1}>0$ such that $x_{1}(t) \geq m_{3}$. This completes the proof. 
(a)

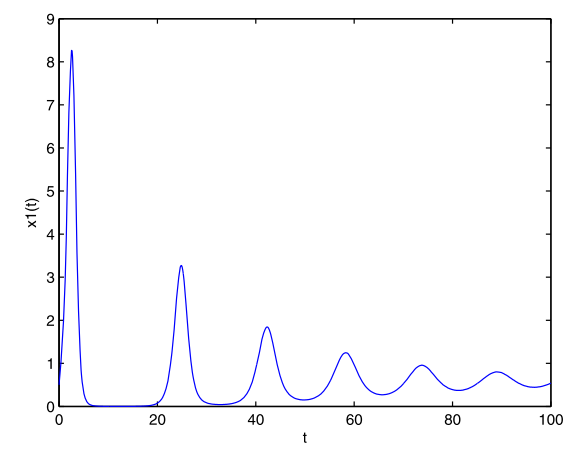

(c)

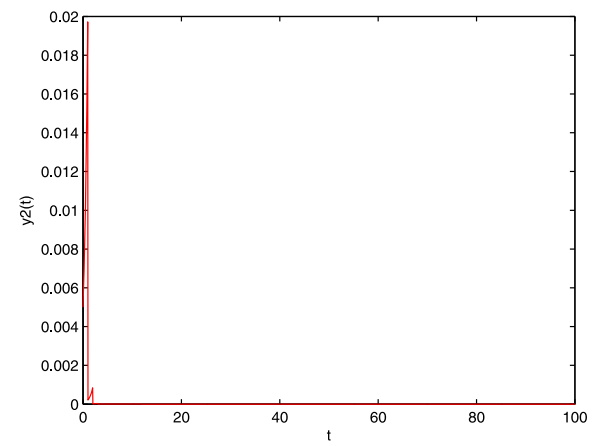

(b)

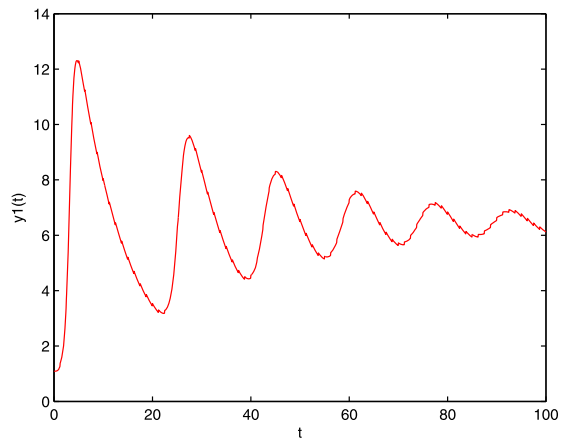

(d)

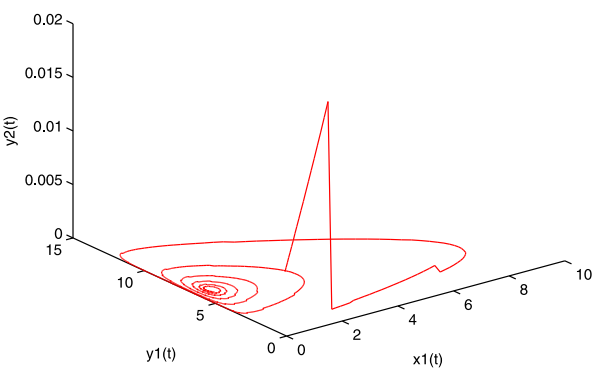

Figure 6 Predator $\left(y_{2}(t)\right)$ extinction and $x_{1}(t)-y_{1}(t)$ permanence of System $(2.1)$ with $x_{1}(0)=0.5$, $y_{1}(0)=0.5, y_{2}(0)=0.5, a_{1}=2, b_{1}=0.1, a_{2}=1.5, b_{2}=0.21, \beta_{1}=0.3, k_{1}=0.5, \mu=0.1, d_{1}=0.1, \tau=1$, $I=\mathbf{0 . 2 5}, \boldsymbol{D}=\mathbf{0 . 9 9}$. (a) Time-series of $x_{1}(t) ;(\mathbf{b})$ time-series of $y_{1}(t) ;(\mathbf{c})$ time-series of $y_{2}(t)$; (d) the phase portrait of predator $\left(y_{2}(t)\right)$ extinction and $x_{1}(t)-y_{1}(t)$ permanence of System (2.1).

\section{Discussion}

In this paper, we establish a predator-prey model with impulsive diffusion and releasing on predator population. This predator-prey model for two regions, which are connected by diffusion of the predator population, portrays the evolution of the population. We prove that all solutions of the investigated system are uniformly ultimately bounded. From Theorem 4.1 , the prey-extinction periodic solution $\left(0, \widehat{y_{1}(t)}, 0\right)$ of System $(2.1)$ is globally asymptotically stable. From Theorem 4.2 , the prey-extinction periodic solution $\left(0, \widetilde{y_{1}(t)}, \widetilde{y_{2}(t)}\right)$ of System (2.1) is globally asymptotically stable. From Theorem 4.4 , System (2.1) is permanent. It is assumed that $x_{1}(0)=0.5, y_{1}(0)=0.5, y_{2}(0)=0.5, a_{1}=2.4, b_{1}=0.1, a_{2}=1.5$, $b_{2}=0.21, \beta_{1}=0.3, k_{1}=0.5, \mu=0.86, d_{1}=0.1, \tau=1, l=0.25, D=0.78$; the conditions (4.1) and (4.2) are obviously satisfied, then the prey-extinction periodic solution $\left(0, \widehat{y_{1}(t)}, 0\right)$ of System (2.1) is globally asymptotically stable (see Figure 3 ). It is assumed that $x_{1}(0)=0.5$, $y_{1}(0)=0.5, y_{2}(0)=0.5, a_{1}=2.4, b_{1}=0.1, a_{2}=1.5, b_{2}=0.21, \beta_{1}=0.3, k_{1}=0.5, \mu=0.86$, $d_{1}=0.1, \tau=1, l=0.25, D=0.6$; the conditions (4.9) and (4.10) are obviously satisfied, then the prey-extinction periodic solution $\left(0, \widetilde{y_{1}(t)}, \widetilde{y_{2}(t)}\right)$ of System $(2.1)$ is globally asymptotically stable (see Figure 4). It is also assumed that $x_{1}(0)=0.5, y_{1}(0)=0.5, y_{2}(0)=0.5, a_{1}=4$, $b_{1}=0.1, a_{2}=4, b_{2}=0.21, \beta_{1}=0.3, k_{1}=0.1, \mu=0.01, d_{1}=0.1, \tau=1, l=0.25, D=0.01$; the conditions (4.11) and (4.12) are obviously satisfied, then System (2.1) is permanent (see Figure 5). It is also assumed that $x_{1}(0)=0.5, y_{1}(0)=0.5, y_{2}(0)=0.5, a_{1}=2, b_{1}=0.1, a_{2}=1.5$, 
$b_{2}=0.21, \beta_{1}=0.3, k_{1}=0.5, \mu=0.1, d_{1}=0.1, \tau=1, l=0.25, D=0.99 ;$ predator $\left(y_{2}(t)\right)$ will go into extinction and $x_{1}(t)-y_{1}(t)$ will be permanent in System (2.1) (see Figure 6).

From the simulations, we can guess that there exist three controlling thresholds with $D$. It is always assumed that $0<D^{*}<D^{* *}<D^{* * *}<1$. If $0<D<D^{*}$ holds, System (2.1) is permanent. If $D^{*}<D<D^{* *}$ holds, the prey-extinction periodic solution $\left(0, \widetilde{y_{1}(t)}, \widetilde{y_{2}(t)}\right)$ of System (2.1) is globally asymptotically stable. If $D^{* *}<D<D^{* * *}$ holds, the prey-extinction periodic solution $\left(0, \widehat{y_{1}(t)}, 0\right)$ of System $(2.1)$ is globally asymptotically stable. If $D^{* * *}<D<1$ holds, the predator $y_{2}(t)$ will go into extinction, prey $x_{1}(t)$ and predator $y_{1}(t)$ will be permanent. We can discuss parameter $\mu$ similar to parameter $D$. We discover that the diffusive rate of the predator population plays an important role in pest management. We conclude that the impulsive diffusion and the released predator provide reliable tactic bases for pest management.

\section{Competing interests}

The authors declare that they have no competing interests.

\section{Authors' contributions}

JJ carried out the main part of this article, LS corrected the manuscript, SH, LM, and YJ brought forward some suggestions on this article. All authors have read and approved the final manuscript.

\section{Author details}

'School of Mathematics and Statistics, Guizhou University of Finance and Economics, Guiyang, 550004, P.R. China. ${ }^{2}$ School of Continuous Education, Guizhou University of Finance and Economics, Guiyang, 550004, P.R. China. ${ }^{3}$ College of Mathematics and Information Science, Anshan Normal University, Anshan, 114007, P.R. China.

\section{Acknowledgements}

Article is supported by the National Natural Science Foundation of China $(11361014,10961008)$, and the project of high level creative talents in Guizhou province (No. 20164035).

Received: 27 January 2016 Accepted: 28 April 2016 Published online: 08 June 2016

\section{References}

1. Caltagirone, LE, Doutt, RL: The history of the vedalia beetle importation to California and its impact on the development of biological control. Annu. Rev. Entomol. 34, 1-16 (1989)

2. DeBach, P: Biological Control of Insect Pests and Weeds. Rheinhold, New York (1964)

3. DeBach, P, Rosen, D: Biological Control by Natural Enemies, 2nd edn. Cambridge University Press, Cambridge (1991)

4. Barclay, HJ: Models for pest control using predator release, habitat management and pesticide release in combination. J. Appl. Ecol. 19, 337-348 (1982)

5. Murray, JD: Mathematical Biology. Springer, Berlin (1989)

6. Freedman, HJ: Graphical stability, enrichment, and pest control by a natural enemy. Math. Biosci. 31, $207-225$ (1976)

7. Grasman, J, Van Herwaarden, OA, et al.: A two-component model of host-parasitoid interactions: determination of the size of inundative releases of parasitoids in biological pest control. Math. Biosci. 169, 207-216 (2001)

8. Liu, X, Chen, L: Compex dynamics of Holling type II Lotka-Volterra predator-prey system with impulsive perturbations on the predator. Chaos Solitons Fractals 16, 311-320 (2003)

9. Jiao, J, Meng, X, Chen, L: A stage-structured Holling mass defence predator-prey model with impulsive perturbations on predators. Appl. Math. Comput. 189, 1448-1458 (2007)

10. Levin, SA: Dispersion and population interaction. Am. Nat. 108, 207-228 (1994)

11. Allen, LJS: Persistence and extinction in single-species reaction-diffusion models. Bull. Math. Biol. 45, $209-227$ (1983)

12. Song, $X Y$, Chen, LS: Uniform persistence and global attractivity for nonautonomous competitive systems with dispersion. J. Syst. Sci. Complex. 15, 307-314 (2002)

13. Cui, JA, Chen, LS: Permanence and extinction in logistic and Lotka-Volterra system with diffusion. J. Math. Anal. Appl. $258,512-535(2001)$

14. Beretta, E, Takeuchi, Y: Global asymptotic stability of Lotka-Volterra diffusion models with continuous time delays. SIAM J. Appl. Math. 48, 627-651 (1998)

15. Beretta, E, Takeuchi, Y: Global stability of single species diffusion Volterra models with continuous time delays. Bull. Math. Biol. 49, 431-448 (1987)

16. Freedman, HI, Takeuchi, Y: Global stability and predator dynamics in a model of prey dispersal in a patchy environment. Nonlinear Anal. 13, 993-1002 (1989)

17. Freedman, $\mathrm{HI}$ : Single species migration in two habitats: persistence and extinction. Math. Model. 8, 778-780 (1987)

18. Freedman, HI, Rai, B, Waltman, P: Mathematical models of population interactions with dispersal ii: differential survival in a change of habitat. J. Math. Anal. Appl. 115, 140-154 (1986)

19. Freedman, HI, Takeuchi, Y: Predator survival versus extinction as a function of dispersal in a predator-prey model with patchy environment. Appl. Anal. 31, 247-266 (1989)

20. Hui, J, Chen, L-s: A single species model with impulsive diffusion. Acta Math. Appl. Sinica (Engl. Ser.) 21, 43-48 (2005) 
21. Bainov, D, Simeonov, P: Impulsive Differential Equations: Periodic Solution and Applications. Longman, London (1993)

22. Jiao, J, et al.: Dynamics of a stage-structured predator-prey model with prey impulsively diffusing between two patches. Nonlinear Anal., Real World Appl. 11, 2748-2756 (2010)

23. Meng, $X$, Chen, L: Permanence and global stability in an impulsive Lotka-Volterra $N$-species competitive system with both discrete delays and continuous delays. Int. J. Biomath. 1, 179-196 (2008)

24. Jiao, J, et al.: An appropriate pest management SI model with biological and chemical control concern. Appl. Math. Comput. 196, 285-293 (2008)

25. Jiao, J, Chen, L: Global attractivity of a stage-structure variable coefficients predator-prey system with time delay and impulsive perturbations on predators. Int. J. Biomath. 1, 197-208 (2008)

26. Jiao, J, et al.: A delayed stage-structured predator-prey model with impulsive stocking on prey and continuous harvesting on predator. Appl. Math. Comput. 195, 316-325 (2008)

27. Jiao, J, et al.: Analysis of a stage-structured predator-prey system with birth pulse and impulsive harvesting at different moments. Nonlinear Anal., Real World Appl. 12, 2232-2244 (2011)

28. Lakshmikantham, V: Theory of Impulsive Differential Equations. World Scientific, Singapore (1989)

29. Chen, L, Meng, X, Jiao, J: Biological Dynamics. Science, Beijing (2009) (in Chinese)

30. Gakkhar, S, Negi, K: Pulse vaccination in SIRS epidemic model with non-monotonic incidence rate. Chaos Solitons Fractals 35, 626-638 (2008)

31. Zhou, Y, Liu, H: Stability of periodic solutions for an SIS model with pulse vaccination. Math. Comput. Model. 38 299-308 (2003)

32. Stone, L, Shulgin, B, Agur, Z: Theoretical examination of the pulse vaccination policy in the SIR epidemic models. Math. Comput. Model. 31, 207-215 (2000)

33. Gao, S, Chen, L, Nieto, JJ, Torres, A: Analysis of a delayed epidemic model with pulse vaccination and saturation incidence. Vaccine 24,6037-6045 (2006)

34. Jury, EL: Inners and Stability of Dynamics System. Wiley, New York (1974)

\section{Submit your manuscript to a SpringerOpen ${ }^{\circ}$ journal and benefit from:}

- Convenient online submission

- Rigorous peer review

Immediate publication on acceptance

- Open access: articles freely available online

- High visibility within the field

- Retaining the copyright to your article 